Moroccan J. of Pure and Appl. Anal. (MJPAA)

Volume 6(1), 2020, Pages 16-33

ISSN: Online 2351-8227 - Print 2605-6364

DOI: $10.2478 / \mathrm{mjpaa}-2020-0002$

\title{
Existence of a weak bounded solution for nonlinear degenerate elliptic equations in Musielak-Orlicz spaces
}

\author{
M. BOURAHMA ${ }^{1}$, J. BENNOUNA ${ }^{2}$ AND M. EL MOUMNI ${ }^{3}$
}

АвSTRACT. In this paper, we show the existence of solutions for the nonlinear elliptic equations of the form

$$
\left\{\begin{array}{l}
-\operatorname{div} a(x, u, \nabla u)=f, \\
u \in W_{0}^{1} L_{\varphi}(\Omega) \cap L^{\infty}(\Omega),
\end{array}\right.
$$

where $a(x, s, \xi) \cdot \xi \geq \bar{\varphi}_{x}^{-1}(\varphi(x, h(|s|))) \varphi(x,|\xi|)$ and $\left.\left.h: \mathbb{R}^{+} \rightarrow\right] 0,1\right]$ is a continuous decreasing function with unbounded primitive. The second term $f$ belongs to $L^{N}(\Omega)$ or to $L^{m}(\Omega)$, with $m=\frac{r N}{r+1}$ for some $r>0$ and $\varphi$ is a Musielak function satisfying the $\Delta_{2}$-condition.

Mathematics Subject Classification (2010): Primary; 35J70, 35J87, Secondary; $46 E 30$.

Key words and phrases: Elliptic equations, Musielak-Orlicz-Sobolev spaces, $L^{\infty}$-estimates,

Bounded solutions.

\section{Introduction}

In recent years, there has been an increasing interest in the analysis of various mathematical problems in the spaces generated by modular functions. These problems have many considerations in applications. Classical Sobolev-spaces, variable exponent Sobolev-spaces and OrliczSobolev spaces are particular types of Musielak Sobolev-spaces which are defined by a more

Received : 20 November 2019 - Accepted: 26 February 2020.

(C) This article is published with open access by Sidi Mohamed Ben Abdallah University.

The Author(s):

${ }^{1}$ Department of Mathematics, laboratory LAMA, Faculty of Sciences Dhar El Mahraz, Sidi Mohammed Ben Abdallah University, PB 1796 Fez-Atlas, Fez, Morocco, e-mail: mohamedbourahma@gmail.com (Corresponding Author).

${ }^{2}$ Department of Mathematics, Faculty of Sciences Dhar El Mahraz, Sidi Mohammed Ben Abdallah University, PB 1796 Fez-Atlas, Fez, Morocco, e-mail: jbennouna@hotmail.com

${ }^{3}$ Department of Mathematics, Faculty of Sciences El Jadida, Chouaib Doukkali University, P.O.Box 20, 24000 El Jadida, Morocco,e-mail: mostafaelmoumni@gmail.com . 
general function $\varphi(x, t)$ which depends also on the space variable. This $x$-dependance creates some difficulties in dealing with these problems. Good news arising from the functional analysis of the setting concerning the density results of smooth functions in the modular topology in Musielak-Orlicz-Sobolev spaces under sufficient assumptions (see [2]) on the Musielak function $\varphi(x, t)$, namely,

$\left(\Phi_{1}\right)$ The function $\varphi$ (resp. $\bar{\varphi}$ ) is locally integrable, that is for any constant number $c>0$ and for any compact set $K \subset \Omega$ we have $\int_{K} \varphi(x, c) d x<\infty$.

$\left(\Phi_{2}\right)$ There exists a function $\varphi_{0}:[0,1 / 2] \times[0, \infty) \longrightarrow[0, \infty)$ such that $\varphi_{0}(., s)$ and $\varphi_{0}(x,$.$) are$ nondecreasing functions and for all $x, y \in \bar{\Omega}$ with $|x-y|<\frac{1}{2}$ and for any constant $c>0$

$$
\varphi(x, s) \leq \varphi_{0}(|x-y|, s) \varphi(y, s) \text { with } \limsup _{\varepsilon \rightarrow 0^{+}} \varphi_{0}\left(\varepsilon, c \varepsilon^{-N}\right)<\infty .
$$

These new results are necessary to study the partial differential equations in Musielak setting. Let $\Omega$ be a bounded subset of $\mathbb{R}^{N}, N \geq 2$ and $\varphi$ is a Musielak function satisfying the $\triangle_{2}$-condition. Let $A: D(A) \subset W_{0}^{1} L_{\varphi}(\Omega) \rightarrow W^{-1} L_{\bar{\varphi}}(\Omega)$ be an operator of Leray-Lions type of the form:

$$
\mathrm{A}(u):=-\operatorname{div} a(x, u, \nabla u) .
$$

In this paper we prove an existence result of bounded solutions in the setting of Musielak spaces for the elliptic problem

$$
\begin{cases}-\operatorname{div} a(x, u, \nabla u)=f & \text { in } \Omega, \\ u=0 & \text { on } \partial \Omega,\end{cases}
$$

where the vector $a(\cdot, \cdot, \cdot)$ satisfies

$$
a(x, s, \xi) \cdot \xi \geq \bar{\varphi}_{x}^{-1}(\varphi(x, h(|s|))) \varphi(x,|\xi|) .
$$

Existence of solutions for problem (1.1) has been vigorously studied in the special cases mentioned above, see for instance [4, 6, 9]. And then in [19], Youssfi has proved the existence of bounded solutions for problem (1.1) in Orlicz-Sobolev spaces $W_{0}^{1} L_{M}(\Omega)$ under the condition

$$
a(x, s, \xi) \cdot \xi \geq \bar{M}^{-1}(M(h(|s|))) M(|\xi|) .
$$

Our purpose in this study, is to prove the existence of weak bounded solution for problem (1.1) in the setting of Musielak-Orlicz-Sobolev spaces under a more general condition than (1.3). Our result generalizes the work in [19].

This paper is organized as follows, in section 2, we recall some well-known preliminaries, results and properties of Musielak-Orlicz-Sobolev spaces. Section 3 is devoted to basic assumptions and problem setting. Finally, we give the proof of the main result in section 4 .

\section{Preliminaries}

In this section we list briefly some definitions and facts about Musielak-Orlicz-Sobolev spaces. For further definitions and properties we refer the reader to $[8,16]$. 
2.1. Musielak-Orlicz function. Let $\Omega$ be an open subset of $\mathbb{R}^{N}, N \geq 2$ and let $\varphi$ be real-valued function defined in $\Omega \times \mathbb{R}^{+}$and satisfying the following conditions

(a) $\varphi(x,$.$) is an \mathrm{N}$-function, i.e., convex, nondecreasing, continuous, $\varphi(x, 0)=0, \varphi(x, t)>0$ for all $t>0$ and

$$
\begin{array}{ll}
\limsup _{t \rightarrow 0} \frac{\varphi(x, t)}{t}=0 & \text { for almost all } x \in \Omega, \\
\lim _{t \rightarrow \infty} \inf _{x \in \Omega} \frac{\varphi(x, t)}{t}=\infty & \text { for almost all } x \in \Omega .
\end{array}
$$

(b) $\varphi(., t)$ is a measurable function.

A function $\varphi(x, t)$, which satisfies the conditions $(a)$ and $(b)$, is called a Musielak-Orlicz function. For a Musielak-Orlicz function $\varphi(x, t)$ we put $\varphi_{x}(t)=\varphi(x, t)$ and we associate its nonnegative reciprocal function with respect to $t$ and $\varphi_{x}^{-1}$ that is,

$$
\varphi_{x}^{-1}(\varphi(x, t))=\varphi\left(x, \varphi_{x}^{-1}(t)\right)=t .
$$

For any two Musielak-Orlicz functions $\varphi$ and $\gamma$ we introduce the following ordering:

(c) If there exists two positive constants $c$ and $T$ such that for almost all $x \in \Omega$

$$
\varphi(x, t) \leq \gamma(x, c t) \text { for } t \geq T,
$$

then we write $\varphi \prec \gamma$ and we say that $\gamma$ dominates $\varphi$ globally if $T=0$ and near infinity if $T>0$.

(d) If for every positive constant $c$ and almost everywhere $x \in \Omega$ we have

$$
\lim _{t \rightarrow 0}\left(\sup _{x \in \Omega} \frac{\varphi(x, c t)}{\gamma(x, t)}\right)=0 \text { or } \lim _{t \rightarrow \infty}\left(\sup _{x \in \Omega} \frac{\varphi(x, c t)}{\gamma(x, t)}\right)=0,
$$

then we write $\varphi \prec \prec \gamma$ at 0 or near $\infty$ respectively, and we say that $\varphi$ increases essentially more slowly than $\gamma$ at 0 or near $\infty$ respectively.

We recall that the Musielak function $\varphi$ is said to satisfy the $\Delta_{2}$-condition (or doubling) if for some $k>0$, and a non-negative function $c$, integrable on $\Omega$, we have

$$
\varphi(x, 2 t) \leq k \varphi(x, t)+c(x) \text { for all } x \in \Omega \text { and all } t \geq 0 \text {. }
$$

2.2. Musielak-Orlicz-Sobolev spaces. For a Musielak function $\varphi$ and a measurable function $u: \Omega \rightarrow \mathbb{R}$ we define the functional

$$
\varrho_{\varphi, \Omega}(u)=\int_{\Omega} \varphi(x,|u(x)|) d x
$$

The set $K_{\varphi}(\Omega)=\left\{u: \Omega \rightarrow \mathbb{R}\right.$ measurable $\left.: \varrho_{\varphi, \Omega}(u)<\infty\right\}$ is called the Musielak class (or the Musielak-Orlicz class or generalized Orlicz class). The Musielak space (or Musielak-Orlicz space or generalized Orlicz space) $L_{\varphi}(\Omega)$ is the vector space generated by $K_{\varphi}(\Omega)$, that is the smallest linear space containing the set $K_{\varphi}(\Omega)$. Equivalently

$$
L_{\varphi}(\Omega)=\left\{u: \Omega \rightarrow \mathbb{R} \text { measurable }: \varrho_{\varphi, \Omega}\left(\frac{u}{\lambda}\right)<\infty \text { for some } \lambda>0\right\} .
$$


For a Musielak function $\varphi$ we put

$$
\bar{\varphi}(x, s)=\sup _{t \geq 0}\{s t-\varphi(x, t)\} .
$$

$\bar{\varphi}$ is called the Musielak function complementary to $\varphi$ (or conjugate of $\varphi$ ) in the sense of Young with respect to $\mathrm{s}$.

we say that a sequence of function $u_{n} \in L_{\varphi}(\Omega)$ is modular convergent to $u \in L_{\varphi}(\Omega)$ if there exists a constant $\lambda>0$ such that

$$
\lim _{n \rightarrow \infty} \varrho_{\varphi, \Omega}\left(\frac{u_{n}-u}{\lambda}\right)=0 .
$$

In the space $L_{\varphi}(\Omega)$ we can define two norms, the first is called the Luxemburg norm, that is

$$
\|u\|_{\varphi, \Omega}=\inf \left\{\lambda>0: \int_{\Omega} \varphi\left(x, \frac{|u(x)|}{\lambda} d x \leq 1\right\}\right.
$$

and the second so-called the Orlicz norm, that is

$$
\left|\left\|u\left|\|_{\varphi, \Omega}=\sup _{\|v\|_{\bar{\varphi}} \leq 1} \int_{\Omega}\right| u(x) v(x) \mid d x,\right.\right.
$$

where $\bar{\varphi}$ is the Musielak function complementary to $\varphi$. These two norms are equivalent and we have a Musielak class $K_{\varphi}(\Omega)$ is a convex subset of the Musielak space $L_{\varphi}(\Omega)$.

The closure in $L_{\varphi}(\Omega)$ of the set of bounded measurable functions with compact support in $\bar{\Omega}$ is denoted by $E_{\varphi}(\Omega)$. It is a separable space and if $\varphi \in \triangle_{2}$, we have $\left(E_{\bar{\varphi}}(\Omega)\right)^{*}=L_{\varphi}(\Omega)$. We have $E_{\varphi}(\Omega)=K_{\varphi}(\Omega)$ if and only if $K_{\varphi}(\Omega)=L_{\varphi}(\Omega)$ if and only if $\varphi$ satisfy the $\Delta_{2}$-condition for large values of $t$ or for all values of $t$, according to whether $\Omega$ has finite measure or not. We define

$$
\begin{aligned}
& W^{1} L_{\varphi}(\Omega)=\left\{u \in L_{\varphi}(\Omega): D^{\alpha} \in L_{\varphi}(\Omega), \forall|\alpha| \leq 1\right\} \\
& W^{1} E_{\varphi}(\Omega)=\left\{u \in E_{\varphi}(\Omega): D^{\alpha} \in E_{\varphi}(\Omega), \forall|\alpha| \leq 1\right\}
\end{aligned}
$$

where $\alpha=\left(\alpha_{1}, \ldots, \alpha_{N}\right),|\alpha|=\left|\alpha_{1}\right|+\ldots+\left|\alpha_{N}\right|$ and $D^{\alpha} u$ denote the distributional derivatives. The space $W^{1} L_{\varphi}(\Omega)$ is called the Musielak-Sobolev space. For $u \in W^{1} L_{\varphi}(\Omega)$, let

$$
\bar{\varrho}_{\varphi, \Omega}(u)=\sum_{|\alpha| \leq 1} \varrho_{\varphi, \Omega}\left(D^{\alpha} u\right) \text { and }\|u\|_{\varphi, \Omega}^{1}=\inf \left\{\lambda>0: \bar{\varrho}_{\varphi, \Omega}\left(\frac{u}{\lambda}\right) \leq 1\right\}
$$

these functionals are convex modular and a norm on $W^{1} L_{\varphi}(\Omega)$ respectively. The pair $\left\langle W^{1} L_{\varphi}(\Omega),\|u\|_{\varphi, \Omega}^{1}\right\rangle$ is a Banach space if $\varphi$ satisfy the following condition

$$
\text { there exists a constant } c>0 \text { such that } \inf _{x \in \Omega} \varphi(x, 1)>c \text {. }
$$

The space $W^{1} L_{\varphi}(\Omega)$ is identified to a subspace of the product $\Pi_{|\alpha| \leq 1} L_{\varphi}(\Omega)=\Pi L_{\varphi}$; this subspace is $\sigma\left(\Pi L_{\varphi}, \Pi E_{\bar{\varphi}}\right)$ closed.

We denote by $\mathfrak{D}(\Omega)$ the Schwartz space of infinitely smooth function with compact support in $\Omega$ and by $\mathfrak{D}(\bar{\Omega})$ the restriction of $\mathfrak{D}\left(\mathbb{R}^{N}\right)$ on $\Omega$. We have $\mathfrak{D}(\Omega) \subset E_{\varphi}(\Omega)$ and $\left(E_{\varphi}(\Omega)\right)^{*}$ is 
isomorphic to $L_{\varphi}(\Omega)$, then the space $W_{0}^{1} L_{\varphi}(\Omega)$ is well defined as the $\sigma\left(\Pi L_{\varphi}, \Pi E_{\bar{\varphi}}\right)$ closure of $\mathfrak{D}(\Omega)$ in $W^{1} L_{\varphi}(\Omega)$ and the space $W_{0}^{1} E_{\varphi}(\Omega)$ as the (norm) closure of the Schwarz space $\mathfrak{D}(\Omega)$ in $W^{1} L_{\varphi}(\Omega)$.

For two complementary Musielak functions $\varphi$ and $\bar{\varphi}$ we have

i)The Young inequality:

$$
t s \leq \varphi(x, t)+\bar{\varphi}(x, s) \text { for all } t, s \geq 0, x \in \Omega .
$$

ii)The Hölder inequality:

$$
\left|\int_{\Omega} u(x) v(x) d x\right| \leq 2\|u\|_{\varphi, \Omega}\|v\|_{\bar{\varphi}, \Omega} \text {, for all } u \in L_{\varphi}(\Omega), v \in L_{\bar{\varphi}}(\Omega) .
$$

We say that a sequence of function $u_{n}$ converges to $u$ for the modular convergence in $W^{1} L_{\varphi}(\Omega)$ (respectively in $W_{0}^{1} L_{\varphi}(\Omega)$ ) if we have

$$
\lim _{n \rightarrow \infty} \bar{\varrho}_{\varphi, \Omega}\left(\frac{u_{n}-u}{\lambda}\right)=0, \text { for some } \lambda>0 \text {. }
$$

Define the following space of distributions

$$
W^{-1} L_{\bar{\varphi}}(\Omega)=\left\{f \in \mathfrak{D}^{\prime}(\Omega): f=\sum_{|\alpha| \leq 1}(-1)^{|\alpha|} D^{\alpha} f_{\alpha} \text { where } f_{\alpha} \in L_{\bar{\varphi}}(\Omega)\right\}
$$

and

$$
W^{-1} E_{\bar{\varphi}}(\Omega)=\left\{f \in \mathfrak{D}^{\prime}(\Omega): f=\sum_{|\alpha| \leq 1}(-1)^{|\alpha|} D^{\alpha} f_{\alpha} \text { where } f_{\alpha} \in E_{\bar{\varphi}}(\Omega)\right\} .
$$

\subsection{Some technical lemmas.}

Lemma 2.1. [2] Assume that $\Omega$ satisfies the segment property and $\varphi$ is Musielak function satisfies assumptions $\left(\Phi_{1}\right)$ and $\left(\Phi_{2}\right)$ and $\bar{\varphi}$ satisfies $\left(\Phi_{1}\right)$, then $\mathfrak{D}(\Omega)$ is dense in $L_{\varphi}(\Omega)$ with respect to the modular topology, $\mathfrak{D}(\Omega)$ is dense in $W_{0}^{1} L_{\varphi}(\Omega)$ for the modular convergence and $\mathfrak{D}(\bar{\Omega})$ is dense in $W^{1} L_{\varphi}(\Omega)$ for the modular convergence.

Consequently, the action of a distribution in $W^{-1} L_{\bar{\varphi}}(\Omega)$ on an element $u$ of $W_{0}^{1} L_{\varphi}(\Omega)$ is well defined.

Recall that an open domain $\Omega \subset \mathbb{R}^{N}$ has the segment property (see [13]) if there exist a locally finite open covering $O_{i}$ of the boundary $\partial \Omega$ of $\Omega$ and a corresponding vectors $y_{i}$ such that if $x \in \bar{\Omega} \cap O_{i}$ for some $i$, then $x+t y_{i} \in \Omega$ for $0<t<1$.

Lemma 2.2. [5](The Nemytskii operator) Let $\Omega$ be an open subset of $\mathbb{R}^{N}$ with finite measure and let $\varphi$ and $\psi$ be two Musielak functions. Let $f: \Omega \times \mathbb{R}^{p_{1}} \rightarrow \mathbb{R}^{p_{2}}$ be a Caratheodory function such that

$$
|f(x, s)| \leq c(x)+k_{1} \psi_{x}^{-1} \varphi\left(x, k_{2}|s|\right),
$$

for almost every $x \in \Omega$ and all $s \in \mathbb{R}^{p_{1}}$, where $k_{1}, k_{2}$ are real positive constant and $c \in E_{\psi}(\Omega)$. Then the Nemytskii operator $N_{f}$, defined by $N_{f}(u)(x)=f(x, u(x))$ is continuous from $\left(\mathbf{P}\left(E_{\varphi}(\Omega), \frac{1}{k_{2}}\right)\right)^{p_{1}}=$ $\Pi\left\{u \in L_{\varphi}(\Omega): d\left(u, E_{\varphi}(\Omega)<\frac{1}{k_{2}}\right\}\right.$ into $\left(L_{\psi}\right)^{p_{2}}$ for the modular convergence. 
Furthermore, if $c \in E_{\gamma}(\Omega)$ and $\gamma \prec \prec \psi$ then $N_{f}$ is strongly continuous from $\left(\mathbf{P}\left(E_{\varphi}(\Omega), \frac{1}{k_{2}}\right)\right)^{p_{1}}$ into $\left(E_{\gamma}(\Omega)\right)^{p_{2}}$.

Lemma 2.3. If $f_{n} \subset L^{1}(\Omega)$ with $f_{n} \rightarrow f \in L^{1}(\Omega)$ a.e. in $\Omega, f_{n}, f \geq 0$ a.e. in $\Omega$ and $\int_{\Omega} f_{n}(x) d x \rightarrow$ $\int_{\Omega} f(x) d x$, then $f_{n} \rightarrow f$ in $L^{1}(\Omega)$.

2.4. Decreasing rearrangement. We recall the definition of decreasing rearrangement of a realvalued measurable function $u$ in a measurable subset $\Omega$ of $\mathbb{R}^{N}$ having finite measure. Let $|E|$ stands the Lebesgue measure of a subset $E$ of $\Omega$. The distribution function of $u$, denoted by $\mu_{u}$, is a map which informs about the content of level sets of $u$, that is

$$
\mu_{u}(t)=|\{x \in \Omega:|u(x)|>t\}|, \quad t \geq 0 .
$$

The decreasing rearrangement of $u$ is defined as the generalized inverse function of $\mu_{u}$, that is the function $u^{*}:[0,|\Omega|] \rightarrow[0,+\infty]$, defined as

$$
u^{*}(s)=\inf \left\{t \geq 0: \mu_{u}(t) \leq s\right\}, \quad s \in[0,|\Omega|] .
$$

In other words, $u^{*}$ is the (unique) non-increasing, right-continuous function in $[0,+\infty)$ equidistributed with $u$. Furthermore, for every $t \geq 0$

$$
u^{*}\left(\mu_{u}(t)\right) \leq t
$$

We also recall that

$$
u^{*}(0)=e s s \sup |u|
$$

\section{Assumptions and main result}

Through this paper $\Omega$ will be a bounded open subset of $\mathbb{R}^{N}, N \geq 2$, satisfying the segment property and $\varphi$ is a doubling Musielak function. There exists an Orlicz function $q$ (see remark 3.1 below) such that

$$
q(t) \leq \varphi(x, t)
$$

Let $A: D(A) \subset W_{0}^{1} L_{\varphi}(\Omega) \rightarrow W^{-1} L_{\bar{\varphi}}(\Omega)$ be the mapping (non-everywhere defined) given by

$$
\mathrm{A} u:=-\operatorname{div} a(x, u, \nabla u),
$$

We are interested, in proving the existence of bounded solutions in the setting of Musielak spaces to the nonlinear problem

$$
\begin{cases}\mathrm{A} u:=-\operatorname{div} a(x, u, \nabla u)=f & \text { in } \Omega, \\ u=0 & \text { on } \partial \Omega .\end{cases}
$$

where $a: \Omega \times \mathbb{R} \times \mathbb{R}^{N} \rightarrow \mathbb{R}^{N}$ is a Caratheodory function satisfying, for almost every $x \in \Omega$ and for all $s \in \mathbb{R}, \xi, \eta \in \mathbb{R}^{N}(\xi \neq \eta)$ the following conditions

$$
a(x, s, \xi) \cdot \xi \geq \bar{\varphi}_{x}^{-1}(\varphi(x, h(|s|))) \varphi(x,|\xi|)
$$


where $h: \mathbb{R} \rightarrow \mathbb{R}_{+}^{*}$ is a continuous decreasing function such that $h(0) \leq 1$ and its primitive $H(s)=\int_{0}^{s} h(t) d t$ is unbounded. there exist a function $a_{0}(x) \in E_{\bar{\varphi}}(\Omega)$ and some positive constants $k_{1}, k_{2}, k_{3}$ and $k_{4}$ and a Musielak function $P \prec \prec \varphi$ such that

$$
\begin{gathered}
|a(x, s, \xi)| \leq a_{0}(x)+k_{1} \bar{P}_{x}^{-1}\left(\varphi\left(x, k_{2}|s|\right)\right)+k_{3} \bar{\varphi}_{x}^{-1}\left(\varphi\left(x, k_{4}|\xi|\right)\right), \\
(a(x, s, \xi)-a(x, s, \eta)) \cdot(\xi-\eta)>0 .
\end{gathered}
$$

For that concerns the right hand side the data $f$, we assume one of the following two assumptions: Either

$$
f \in L^{N}(\Omega),
$$

or

$$
\left\{\begin{array}{r}
f \in L^{m}(\Omega) \text { with } m=\frac{r N}{r+1} \text { for some } r>0 \\
\text { and } \int^{+\infty}\left(\frac{t}{q(t)}\right)^{r} d t<+\infty
\end{array}\right.
$$

Remark 3.1. Notice that, in particular case, in variable exponent spaces when $\varphi(x, t)=t^{p(x)}$, the Orlicz function $q(t)$ plays the role of $t^{p^{-}}$, where $p^{-}=e s s \inf _{x \in \Omega} p(x)$. Moreover, $q(t)$ is an $N$-function satisfying the $\Delta_{2}$-condition, for its construction see ( [14],[11, Lemma A.4]).

Definition 3.1. Let $f \in L^{N}(\Omega)$, we say that a function $u \in W_{0}^{1} L_{\varphi}(\Omega)$ is a weak solution of (3.1), if $a(\cdot, u, \nabla u) \in\left(L_{\bar{\varphi}}(\Omega)\right)^{N}$ and

$$
\int_{\Omega} a(x, u, \nabla u) \cdot \nabla v d x=\int_{\Omega} f v d x
$$

holds for all $v \in \mathfrak{D}(\Omega)$.

Lemma 3.1. Let $\varphi$ be a Musielak function and $q$ is an Orlicz function such that $q(t) \leq \varphi(x, t)$ for all $(x, t) \in \Omega \times \mathbb{R}^{+}$then

$$
\bar{q}^{-1}(q(t)) \leq \bar{\varphi}_{x}^{-1}(\varphi(x, t)) \text { for all }(x, t) \in \Omega \times \mathbb{R}^{+},
$$

where $\bar{q}$ and $\bar{\varphi}$ are the complementary functions of $q$ and $\varphi$ respectively.

Proof. Let $s, t \in \mathbb{R}^{+}$and $x \in \Omega$. We have $q(t) \leq \varphi(x, t)$, then

$$
s t-q(t) \geq s t-\varphi(x, t) \text {. }
$$

Passing to the sup over $t \geq 0$

$$
\sup _{t \geq 0}\{s t-q(t)\} \geq \sup _{t \geq 0}\{s t-\varphi(x, t)\} .
$$

That means

$$
\bar{q}(s) \geq \bar{\varphi}(x, s):=\bar{\varphi}_{x}(s), \text { for all } s \in \mathbb{R}^{+} .
$$

It follows that for all $s \in \mathbb{R}^{+}$,

$$
\bar{q}^{-1}(s) \leq \bar{\varphi}_{x}^{-1}(s)
$$

Taking $s=q(t)$, since $\bar{\varphi}_{x}^{-1}$ is increasing, we have $\forall t \in \mathbb{R}^{+}$,

$$
\bar{q}^{-1}(q(t)) \leq \bar{\varphi}_{x}^{-1}(q(t)) \leq \bar{\varphi}_{x}^{-1}(\varphi(x, t)) .
$$


Remark 3.2. Note that from $\int_{\Omega} \varphi(x, c) d x<\infty$ and the fact that $q(t) \leq \varphi(x, t) \Longleftrightarrow \bar{\varphi}(x, t) \leq \bar{q}(t)$, the assumption $\left(\Phi_{1}\right)$ is automatically verified for $\varphi$ and $\bar{\varphi}$.

The next theorem is the main result of this paper.

Theorem 3.1. Suppose that the assumptions $\left(\Phi_{2}\right),(3.2),(3.3),(3.4)$ and either (3.5) or (3.6) are satisfied and assume that $\int_{\Omega} \varphi(x, c) d x<\infty$ for all constant $c>0$. Then the following problem

$$
\begin{cases}\mathrm{A} u:=-\operatorname{div} a(x, u, \nabla u)=f & \text { in } \Omega, \\ u=0 & \text { on } \partial \Omega .\end{cases}
$$

has at least one weak solution in $W_{0}^{1} L_{\varphi}(\Omega) \cap L^{\infty}(\Omega)$.

\section{Proof of main result.}

The proof of theorem (3.1) is divided into six steps.

Step 1: Approximate problems. Let us define $T_{k}(s)=\max (-k, \min (k, s)), k>0$ and the function $G_{k}(s)=s-T_{k}(s)$. For $n \in \mathbb{N}^{*}$ denoting by $m^{*}$ either $N$ or $m$ according as we assume (3.5) or (3.6), and let $\left\{f_{n}\right\} \subset W^{-1} E_{\bar{\varphi}}(\Omega)$ be a sequence of smooth functions such that

$$
f_{n} \rightarrow f \text { strongly in } L^{m^{*}}(\Omega)
$$

and

consider the operator:

$$
\left\|f_{n}\right\|_{m^{*}} \leq\|f\|_{m^{*}}
$$

$$
A_{n}(u):=-\operatorname{div} a\left(x, T_{n}(u), \nabla u\right)
$$

the operator $A_{n}$ satisfies the conditions of [8], then the approximate problem has at least a solution $u_{n}$ such that

$$
\int_{\Omega} a\left(x, T_{n}\left(u_{n}\right), \nabla u_{n}\right) \cdot \nabla v d x=\int_{\Omega} f_{n} v d x
$$

holds for all $v \in W_{0}^{1} L_{\varphi}(\Omega)$.

\section{Step 2: Preliminary results.}

Lemma 4.1. Let $K(t)=\frac{q(t)}{t}$ and $\mu_{n}(t)=\left|\left\{x \in \Omega:\left|u_{n}(x)\right|>t\right\}\right|$, for all $t>0$. We have for almost every $t>0$ :

$$
h(t) \leq \frac{2 q(1)}{\bar{q}^{-1}(q(1)) N C_{N}^{1 / N}} \frac{-\mu_{n}^{\prime}(t)}{\mu_{n}(t)^{1-\frac{1}{N}}} K^{-1}\left(\frac{\int_{\left\{\left|u_{n}\right|>t\right\}}\left|f_{n}\right| d x}{\bar{q}^{-1}(q(1)) N C_{N}^{1 / N} \mu_{n}(t)^{1-\frac{1}{N}}}\right)
$$

where $C_{N}$ is the measure of the unit ball in $\mathbb{R}^{N}$. 
Proof. Testing by $v=T_{k}\left(G_{t}\left(u_{n}\right)\right)$ in (4.1), one has

$$
\int_{\left\{t<\left|u_{n}\right| \leq t+k\right\}} a\left(x, T_{n}\left(u_{n}\right), \nabla u_{n}\right) \cdot \nabla u_{n} d x \leq k \int_{\left\{\left|u_{n}\right|>t\right\}}\left|f_{n}\right| d x .
$$

Then (3.2) yields

$$
\frac{1}{k} \int_{\left\{t<\left|u_{n}\right| \leq t+k\right\}} \bar{\varphi}_{x}^{-1}\left(\varphi\left(x, h\left(\left|u_{n}\right|\right)\right)\right) \varphi\left(x,\left|\nabla u_{n}\right|\right) d x \leq \int_{\left\{\left|u_{n}\right|>t\right\}}\left|f_{n}\right| d x .
$$

Let $k \rightarrow 0^{+}$we have

$$
-\frac{d}{d t} \int_{\left\{\left|u_{n}\right|>t\right\}} \bar{\varphi}_{x}^{-1}\left(\varphi\left(x, h\left(\left|u_{n}\right|\right)\right)\right) \varphi\left(x,\left|\nabla u_{n}\right|\right) d x \leq \int_{\left\{\left|u_{n}\right|>t\right\}}\left|f_{n}\right| d x,
$$

for almost every $t>0$. We use the properties of the function $Q(t)=\frac{1}{K^{-1}(t)}$ which is decreasing and convex (see [17]). By lemma (3.1), $\varphi\left(x,\left|\nabla u_{n}\right|\right) \geq q\left(\left|\nabla u_{n}\right|\right) \geq 0$ and $\bar{\varphi}_{x}^{-1}\left(\varphi\left(x, h\left(\left|u_{n}\right|\right)\right)\right) \geq$ $\bar{q}^{-1}\left(q\left(h\left(\left|u_{n}\right|\right)\right)\right) \geq 0$, then

$$
\bar{\varphi}_{x}^{-1}\left(\varphi\left(x, h\left(\left|u_{n}\right|\right)\right)\right) \varphi\left(x,\left|\nabla u_{n}\right|\right) \geq \bar{q}^{-1}\left(q\left(h\left(\left|u_{n}\right|\right)\right)\right) q\left(\left|\nabla u_{n}\right|\right)
$$

Using the fact that $Q$ is decreasing and then Jensen's inequality yield

$$
\begin{aligned}
& Q\left(\frac{\int_{\left\{t<\left|u_{n}\right| \leq t+k\right\}} \bar{\varphi}_{x}^{-1}\left(\varphi\left(x, h\left(\left|u_{n}\right|\right)\right)\right) \varphi\left(x,\left|\nabla u_{n}\right|\right) d x}{\int_{\left\{t<\left|u_{n}\right| \leq t+k\right\}} \bar{q}^{-1}\left(q\left(h\left(\left|u_{n}\right|\right)\right)\right)\left|\nabla u_{n}\right| d x}\right) \\
& \leq Q\left(\frac{\int_{\left\{t<\left|u_{n}\right| \leq t+k\right\}} \bar{q}^{-1}\left(q\left(h\left(\left|u_{n}\right|\right)\right)\right) q\left(\left|\nabla u_{n}\right|\right) d x}{\int_{\left\{t<\left|u_{n}\right| \leq t+k\right\}} \bar{q}^{-1}\left(q\left(h\left(\left|u_{n}\right|\right)\right)\right)\left|\nabla u_{n}\right| d x}\right) \\
= & Q\left(\frac{\int_{\left\{t<\left|u_{n}\right| \leq t+k\right\}} K\left(\left|\nabla u_{n}\right|\right) \bar{q}^{-1}\left(q\left(h\left(\left|u_{n}\right|\right)\right)\right)\left|\nabla u_{n}\right| d x}{\int_{\left\{t<\left|u_{n}\right| \leq t+k\right\}} \bar{q}^{-1}\left(q\left(h\left(\left|u_{n}\right|\right)\right)\right)\left|\nabla u_{n}\right| d x}\right) \\
\leq & \frac{\int_{\left\{t<\left|u_{n}\right| \leq t+k\right\}} \bar{q}^{-1}\left(q\left(h\left(\left|u_{n}\right|\right)\right)\right) d x}{\int_{\left\{t<\left|u_{n}\right| \leq t+k\right\}} \bar{q}^{-1}\left(q\left(h\left(\left|u_{n}\right|\right)\right)\right)\left|\nabla u_{n}\right| d x} \\
\leq & \frac{\bar{q}^{-1}(q(h(t)))\left(-\mu_{n}(t+k)+\mu_{n}(t)\right)}{\bar{q}^{-1}(q(h(t+k))) \int_{\left\{t<\left|u_{n}\right| \leq t+k\right\}}\left|\nabla u_{n}\right| d x} .
\end{aligned}
$$


Since $\frac{\bar{q}^{-1}(q(h(t)))}{\bar{q}^{-1}(q(1))} \leq 1$, using the convexity of $Q$ and then letting $k \rightarrow 0^{+}$, we get for almost every $t>0$,

$$
\begin{aligned}
& \frac{\bar{q}^{-1}(q(1))}{\bar{q}^{-1}(q(h(t)))} Q\left(\frac{-\frac{d}{d t} \int_{\left\{\left|u_{n}\right|>t\right\}} \bar{\varphi}_{x}^{-1}\left(\varphi\left(x, h\left(\left|u_{n}\right|\right)\right)\right) \varphi\left(x,\left|\nabla u_{n}\right|\right) d x}{\bar{q}^{-1}(q(1))\left(-\frac{d}{d t} \int_{\left\{\left|u_{n}\right|>t\right\}}\left|\nabla u_{n}\right| d x\right)}\right) \\
& \leq \frac{-\mu_{n}^{\prime}(t)}{-\frac{d}{d t} \int_{\left\{\left|u_{n}\right|>t\right\}}\left|\nabla u_{n}\right| d x} .
\end{aligned}
$$

Now we recall the following inequality from [17]:

$$
-\frac{d}{d t} \int_{\left\{\left|u_{n}\right|>t\right\}}\left|\nabla u_{n}\right| d x \geq N C_{N}^{1 / N} \mu_{n}(t)^{1-\frac{1}{N}} \quad \text { for almost every } t>0 .
$$

Thanks to (4.3), (4.4) and the monotonicity of the function $Q$ we obtain

$$
\begin{aligned}
& \frac{1}{\bar{q}^{-1}(q(h(t)))} \\
& \leq \frac{-\mu_{n}^{\prime}(t)}{\bar{q}^{-1}(q(1)) N C_{N}^{1 / N} \mu_{n}(t)^{1-\frac{1}{N}}} K^{-1}\left(\frac{\int_{\left\{\left|u_{n}\right|>t\right\}}\left|f_{n}\right| d x}{\bar{q}^{-1}(q(1)) N C_{N}^{1 / N} \mu_{n}(t)^{1-\frac{1}{N}}}\right) .
\end{aligned}
$$

Using the inequality in Orlicz spaces (see [19])

$$
q(t) \leq t \bar{q}^{-1}(q(t)) \leq 2 q(t) \text { for all } t \geq 0
$$

and the fact that $0<h(t) \leq 1,(4.2)$ follows.

step 3: Uniform $L^{\infty}$-estimation. From (4.2), using the rearrangement techniques, we prove that there exists a constant $c_{\infty}$ such that

$$
\left\|u_{n}\right\|_{\infty} \leq c_{\infty}
$$

Proof. If we assume (3.5), by Hölder's inequality one has

$$
\int_{\left\{\left|u_{n}\right|>t\right\}}\left|f_{n}\right| d x \leq\|f\|_{N} \mu_{n}(t)^{1-\frac{1}{N}}
$$

Inequality (4.2) becomes

$$
h(t) \leq \frac{2 q(1)\left(-\mu_{n}^{\prime}(t)\right)}{\bar{q}^{-1}(q(1)) N C_{N}^{1} \mu_{n}(t)^{1-\frac{1}{N}}} K^{-1}\left(\frac{c_{0}\|f\|_{N}}{\bar{q}^{-1}(q(1)) N C_{N}^{\frac{1}{N}}}\right) .
$$


Then, integrating between 0 and $s$, we get

$$
\int_{0}^{s} h(t) d t \leq \frac{2 q(1)}{\bar{q}^{-1}(q(1)) N C_{N}^{\frac{1}{N}}} K^{-1}\left(\frac{c_{0}\|f\|_{N}}{\bar{q}^{-1}(q(1)) N C_{N}^{\frac{1}{N}}}\right) \int_{0}^{s} \frac{-\mu_{n}^{\prime}(t)}{\mu_{n}(t)^{1-\frac{1}{N}}} d t .
$$

So, one has

$$
H(s) \leq \frac{2 q(1)}{\bar{q}^{-1}(q(1)) N C_{N}^{\frac{1}{N}}} K^{-1}\left(\frac{c_{0}\|f\|_{N}}{\bar{q}^{-1}(q(1)) N C_{N}^{\frac{1}{N}}}\right) \int_{0}^{s} \frac{-\mu_{n}^{\prime}(t)}{\mu_{n}(t)^{1-\frac{1}{N}}} d t .
$$

Hence, a change of variables yields

$$
H(s) \leq \frac{2 q(1)}{\bar{q}^{-1}(q(1)) N C_{N}^{\frac{1}{N}}} K^{-1}\left(\frac{c_{0}\|f\|_{N}}{\bar{q}^{-1}(q(1)) N C_{N}^{\frac{1}{N}}}\right) \int_{\mu_{n}(s)}^{|\Omega|} \frac{d t}{t^{1-\frac{1}{N}}} .
$$

By (2.1) we get

$$
H\left(u_{n}^{*}(\sigma)\right) \leq \frac{2 q(1)}{\bar{q}^{-1}(q(1)) N C_{N}^{\frac{1}{N}}} K^{-1}\left(\frac{c_{0}\|f\|_{N}}{\bar{q}^{-1}(q(1)) N C_{N}^{\frac{1}{N}}}\right) \int_{\sigma}^{|\Omega|} \frac{d t}{t^{1-\frac{1}{N}}} .
$$

So that

$$
H\left(u_{n}^{*}(0)\right) \leq \frac{2 q(1)|\Omega| \frac{1}{N}}{\bar{q}^{-1}(q(1)) C_{N}^{\frac{1}{N}}} K^{-1}\left(\frac{c_{0}\|f\|_{N}}{\bar{q}^{-1}(q(1)) N C_{N}^{\frac{1}{N}}}\right) .
$$

Thanks to (2.2) and the fact that $\lim _{s \rightarrow+\infty} H(s)=+\infty$, we conclude that the sequence $\left\{u_{n}\right\}$ is uniformly bounded in $L^{\infty}(\Omega)$. Moreover, if we denote by $H^{-1}$ the inverse function of $H$, one has:

$$
\left\|u_{n}\right\|_{\infty} \leq H^{-1}\left(\frac{2 q(1)|\Omega|^{\frac{1}{N}}}{\bar{q}^{-1}(q(1)) C_{N}^{\frac{1}{N}}} K^{-1}\left(\frac{c_{0}\|f\|_{N}}{\bar{q}^{-1}(q(1)) N C_{N}^{\frac{1}{N}}}\right)\right) .
$$

We now assume that (3.6) is filled. Then, using again Hölder's inequality we have

$$
\int_{\left\{\left|u_{n}\right|>t\right\}}\left|f_{n}\right| d x \leq\|f\|_{m} \mu_{n}(t)^{1-\frac{1}{m}}
$$

Inequality (4.2) becomes

$$
h(s) \leq \frac{2 q(1)}{\bar{q}^{-1}(q(1)) N C_{N}^{\frac{1}{N}}} \frac{-\mu_{n}^{\prime}(t)}{\mu_{n}(t)^{1-\frac{1}{N}}} K^{-1}\left(\frac{c_{0}\|f\|_{m}}{\bar{q}^{-1}(q(1)) N C_{N}^{\frac{1}{N}} \mu_{n}(t)^{\frac{1}{m}-\frac{1}{N}}}\right) .
$$

Integrating between 0 and $s$, we get

$$
H(s) \leq \frac{2 q(1)}{\bar{q}^{-1}(q(1)) N C_{N}^{\frac{1}{N}}} \int_{0}^{s} \frac{-\mu_{n}^{\prime}(t)}{\mu_{n}(t)^{1-\frac{1}{N}}} K^{-1}\left(\frac{c_{0}\|f\|_{m}}{\bar{q}^{-1}(q(1)) N C_{N}^{\frac{1}{N}} \mu_{n}(t)^{\frac{1}{m}-\frac{1}{N}}}\right) d t .
$$


Then, a change of variables gives

$$
H(s) \leq \frac{2 q(1)}{\bar{q}^{-1}(q(1)) N C_{N}^{\frac{1}{N}}} \int_{\mu_{n}(s)}^{|\Omega|} K^{-1}\left(\frac{c_{0}\|f\|_{m}}{\bar{q}^{-1}(q(1)) N C_{N}^{\frac{1}{N}} \sigma^{\frac{1}{m}-\frac{1}{N}}}\right) \frac{d \sigma}{\sigma^{1-\frac{1}{N}}} .
$$

By virtue of (2.1) we get

$$
H\left(u_{n}^{*}(\tau)\right) \leq \frac{2 q(1)}{\bar{q}^{-1}(q(1)) N C_{N}^{\frac{1}{N}}} \int_{\tau}^{|\Omega|} K^{-1}\left(\frac{c_{0}\|f\|_{m}}{\bar{q}^{-1}(q(1)) N C_{N}^{\frac{1}{N}} \sigma^{\frac{1}{m}-\frac{1}{N}}}\right) \frac{d \sigma}{\sigma^{1-\frac{1}{N}}} .
$$

Then, by (2.2) we obtain

$$
H\left(\left\|u_{n}\right\|_{\infty}\right) \leq \frac{2 q(1)}{\bar{q}^{-1}(q(1)) N C_{N}^{\frac{1}{N}}} \int_{0}^{|\Omega|} K^{-1}\left(\frac{c_{0}\|f\|_{m}}{\bar{q}^{-1}(q(1)) N C_{N}^{\frac{1}{N}} \sigma^{\frac{1}{m}-\frac{1}{N}}}\right) \frac{d \sigma}{\sigma^{1-\frac{1}{N}}} .
$$

A change of variables gives

$$
H\left(\left\|u_{n}\right\|_{\infty}\right) \leq \frac{2 q(1) c_{0}^{r}\|f\|_{m}^{r}}{\left(\bar{q}^{-1}(q(1))\right)^{r+1} N^{r} C_{N}^{\frac{r+1}{N}}} \int_{\lambda}^{+\infty} r t^{-r-1} K^{-1}(t) d t
$$

where $\lambda=\frac{c_{0}\|f\|_{m}}{\bar{q}^{-1}(q(1)) N C_{N}^{\frac{1}{N}}|\Omega|^{\frac{1}{T N}}}$. Then, by an integration by parts we obtain that

$$
H\left(\left\|u_{n}\right\|_{\infty}\right) \leq \frac{2 q(1) c_{0}^{r}\|f\|_{m}^{r}}{\left(\bar{q}^{-1}(q(1))\right)^{r+1} N^{r} C_{N}^{\frac{r+1}{N}}}\left(\frac{K^{-1}(\lambda)}{\lambda^{r}}+\int_{K^{-1}(\lambda)}^{+\infty}\left(\frac{s}{q(s)}\right)^{r} d s\right) .
$$

The assumption made on $H$ guarantees that the sequence $\left\{u_{n}\right\}$ is uniformly bounded in $L^{\infty}(\Omega)$. Indeed, denoting by $H^{-1}$ the inverse function of $H$, one has

$$
\left\|u_{n}\right\|_{\infty} \leq H^{-1}\left(\frac{2 q(1) c_{0}^{r}\|f\|_{m}^{r}}{\left(\bar{q}^{-1}(q(1))\right)^{r+1} N^{r} C_{N}^{\frac{r+1}{N}}}\left(\frac{K^{-1}(\lambda)}{\lambda^{r}}+\int_{K^{-1}(\lambda)}^{+\infty}\left(\frac{s}{q(s)}\right)^{r} d s\right)\right) .
$$

Consequently, in both cases the sequence $\left\{u_{n}\right\}$ is uniformly bounded in $L^{\infty}(\Omega)$, so that in the sequel, we will denote by $c_{\infty}$ the constant appearing either in (4.6) or in (4.7), that is :

$$
\left\|u_{n}\right\|_{\infty} \leq c_{\infty} .
$$

Step 4: Estimation in $W_{0}^{1} L_{\varphi}(\Omega)$. Now we will obtain an estimate in $W_{0}^{1} L_{\varphi}(\Omega)$ under either (3.5) or (3.6). Let $m^{*}$ denotes either $N$ or $m$ according as we assume (3.5) or (3.6). Testing (4.1) by $u_{n}$, it follows

Then by (3.2) and (4.5), we obtain

$$
\int_{\Omega} a\left(x, T_{n}\left(u_{n}\right), \nabla u_{n}\right) \cdot \nabla u_{n} d x=\int_{\Omega} f_{n} u_{n} d x .
$$

$$
\int_{\Omega} \bar{\varphi}_{x}^{-1}\left(\varphi\left(x, h\left(\left|T_{n}\left(u_{n}\right)\right|\right)\right)\right) \varphi\left(x,\left|\nabla u_{n}\right|\right) d x \leq c_{\infty}\|f\|_{m^{*}}|\Omega|^{1-\frac{1}{m^{*}}} .
$$


By lemma (3.1) we have

$$
\begin{aligned}
\int_{\Omega} \bar{q}^{-1}\left(q\left(h\left(\left|T_{n}\left(u_{n}\right)\right|\right)\right)\right) \varphi\left(x,\left|\nabla u_{n}\right|\right) d x & \leq \int_{\Omega} \bar{\varphi}_{x}^{-1}\left(\varphi\left(x, h\left(\left|T_{n}\left(u_{n}\right)\right|\right)\right)\right) \varphi\left(x,\left|\nabla u_{n}\right|\right) d x \\
& \leq c_{\infty}\|f\|_{m^{*}}|\Omega|^{1-\frac{1}{m^{*}}} .
\end{aligned}
$$

Finally, since the function $h$ is decreasing and $T_{n}\left(u_{n}\right) \leq T_{n}\left(c_{\infty}\right) \leq c_{\infty}$, we get

$$
\int_{\Omega} \varphi\left(x,\left|\nabla u_{n}\right|\right) d x \leq \frac{c_{\infty}\|f\|_{m^{*}}|\Omega|^{1-\frac{1}{m^{*}}}}{\bar{q}^{-1}\left(q\left(h\left(c_{\infty}\right)\right)\right)} .
$$

Which implies, by Poincaré's inequality from [3], that the sequence $\left\{u_{n}\right\}$ is bounded in $W_{0}^{1} L_{\varphi}(\Omega)$. And then, there exists a subsequence of $\left\{u_{n}\right\}$, still denoted by $\left\{u_{n}\right\}$, and a function $u$ in $W_{0}^{1} L_{\varphi}(\Omega)$ such that

$$
u_{n} \rightarrow u \text { in } W_{0}^{1} L_{\varphi}(\Omega) \text { for } \sigma\left(\Pi L_{\varphi}, \Pi E_{\bar{\varphi}}\right)
$$

and the compact embedding gives

$$
u_{n} \rightarrow u \text { in } E_{\varphi}(\Omega) \text { strongly and a.e. in } \Omega \text {. }
$$

Step 5: Almost everywhere convergence of the gradients. The next lemma will be used later, so proving it first.

Lemma 4.2. The sequence $\left\{a\left(x, T_{n}\left(u_{n}\right), \nabla u_{n}\right)\right\}$ is bounded in $\left(L_{\bar{\varphi}}(\Omega)\right)^{N}$.

Proof. We will use the dual norm of $\left(L_{\bar{\varphi}}(\Omega)\right)^{N}$. Let $\phi \in\left(E_{\varphi}(\Omega)\right)^{N}$ such that $\|\phi\|_{\varphi}=1$. By (3.4) we have

$$
\left(a\left(x, T_{n}\left(u_{n}\right), \nabla u_{n}\right)-a\left(x, T_{n}\left(u_{n}\right), \frac{\phi}{k_{4}}\right)\right) \cdot\left(\nabla u_{n}-\frac{\phi}{k_{4}}\right) \geq 0 .
$$

Thanks to (3.3), (4.5), (4.9) and Young's inequality we get

$$
\begin{aligned}
& \int_{\Omega} a\left(x, T_{n}\left(u_{n}\right), \nabla u_{n}\right) \phi d x \\
& \leq k_{4} \int_{\Omega} a\left(x, T_{n}\left(u_{n}\right), \nabla u_{n}\right) \cdot \nabla u_{n} d x-k_{4} \int_{\Omega} a\left(x, T_{n}\left(u_{n}\right), \frac{\phi}{k_{4}}\right) \cdot \nabla u_{n} d x \\
& \quad+\int_{\Omega} a\left(x, T_{n}\left(u_{n}\right), \frac{\phi}{k_{4}}\right) \cdot \phi d x \\
& \leq k_{4} c_{\infty}\|f\|_{m^{*}}|\Omega|^{1-\frac{1}{m^{*}}}+k_{4}\left(1+k_{1}+k_{3}\right) \frac{c_{\infty}\|f\|_{m^{*}}|\Omega|^{1-\frac{1}{m^{*}}}}{\bar{q}^{-1}\left(q\left(h\left(c_{\infty}\right)\right)\right)} \\
& \quad+\left(1+k_{4}\right) \int_{\Omega} \bar{\varphi}\left(x, a_{0}(x)\right) d x+k_{1}\left(1+k_{4}\right) \int_{\Omega} \bar{\varphi}\left(x, \bar{P}_{x}^{-1}\left(\varphi\left(x, k_{2} c_{\infty}\right)\right)\right) d x \\
& \quad+\left(k_{3}\left(1+k_{4}\right)+\left(1+k_{1}+k_{3}\right)\right) \int_{\Omega} \varphi(x, 1) d x .
\end{aligned}
$$

Since $a_{0}(x) \in E_{\bar{\varphi}}(\Omega), \int_{\Omega} \bar{\varphi}\left(x, a_{0}(x)\right) d x<+\infty$ and we have $\int_{\Omega} \varphi(x, 1) d x<+\infty$. For the remaining integral in the last inequality, recall that $P \prec \prec \varphi \Leftrightarrow \bar{\varphi} \prec \prec \bar{P}$, and using the fact 
that

$$
\bar{\varphi} \prec \prec \bar{P} \Rightarrow \forall \varepsilon>0, \exists h \in L^{1}(\Omega): \quad \bar{\varphi}(x, t) \leq \bar{P}(x, \varepsilon t)+h(x) .
$$

Thus, taking $\varepsilon \leq 1$, and since $\bar{P}$ is increasing, we get

$$
\begin{aligned}
\int_{\Omega} \bar{\varphi}\left(x, \bar{P}_{x}^{-1}\left(\varphi\left(x, k_{2} c_{\infty}\right)\right)\right) d x & \leq \int_{\Omega} \bar{P}\left(x, \varepsilon \bar{P}_{x}^{-1}\left(\varphi\left(x, k_{2} c_{\infty}\right)\right)\right) d x+\int_{\Omega} h(x) d x \\
& \leq \int_{\Omega} \bar{P}\left(x, \bar{P}_{x}^{-1}\left(\varphi\left(x, k_{2} c_{\infty}\right)\right)\right) d x+\int_{\Omega} h(x) d x \\
& \leq \int_{\Omega} \varphi\left(x, k_{2} c_{\infty}\right) d x+\int_{\Omega} h(x) d x \\
& <+\infty .
\end{aligned}
$$

This ends the proof.

From (4.5) and (4.11) we obtain that $u \in W_{0}^{1} L_{\varphi}(\Omega) \cap L^{\infty}(\Omega)$, so that by lemma 2.1 there exists a sequence $\left\{v_{j}\right\}$ in $\mathfrak{D}(\Omega)$ such that $v_{j} \rightarrow u$ in $W_{0}^{1} L_{\varphi}(\Omega)$ as $j \rightarrow \infty$ for the modular convergence and almost everywhere in $\Omega$.

For $s>0$, we denote by $\chi_{j}^{s}$ the characteristic function of the set

$$
\Omega_{j}^{s}=\left\{x \in \Omega:\left|\nabla v_{j}(x)\right| \leq s\right\}
$$

and by $\chi^{s}$ the characteristic function of the set $\Omega^{s}=\{x \in \Omega:|\nabla u(x)| \leq s\}$. Testing by $u_{n}-v_{j}$ in (4.1), we obtain

$$
\int_{\Omega} a\left(x, T_{n}\left(u_{n}\right), \nabla u_{n}\right) \cdot\left(\nabla u_{n}-\nabla v_{j}\right) d x=\int_{\Omega} f_{n}\left(u_{n}-v_{j}\right) d x
$$

Denote by $\epsilon_{i}(n, j),(i=0,1, \ldots)$, various sequences of real numbers which tend to 0 when $n$ and $j \rightarrow \infty$, i.e.

$$
\lim _{j \rightarrow \infty} \lim _{n \rightarrow \infty} \epsilon_{i}(n, j)=0 .
$$

For the right-hand side of (4.12), we have

$$
\int_{\Omega} f_{n}\left(u_{n}-v_{j}\right) d x=\epsilon_{0}(n, j) .
$$

The left-hand side of (4.12) is written as

$$
\begin{aligned}
& \int_{\Omega} a\left(x, T_{n}\left(u_{n}\right), \nabla u_{n}\right) \cdot\left(\nabla u_{n}-\nabla v_{j}\right) d x \\
& =\int_{\Omega}\left(a\left(x, T_{n}\left(u_{n}\right), \nabla u_{n}\right)-a\left(x, T_{n}\left(u_{n}\right), \nabla v_{j} x_{j}^{s}\right)\right) \cdot\left(\nabla u_{n}-\nabla v_{j} \chi_{j}^{s}\right) d x \\
& \quad+\int_{\Omega} a\left(x, T_{n}\left(u_{n}\right), \nabla v_{j} \chi_{j}^{s}\right) \cdot\left(\nabla u_{n}-\nabla v_{j} \chi_{j}^{s}\right) d x \\
& \quad-\int_{\Omega \backslash \Omega_{j}^{s}} a\left(x, T_{n}\left(u_{n}\right), \nabla u_{n}\right) \cdot \nabla v_{j} d x
\end{aligned}
$$

We will pass to the limit over $n$ and $j$, for $s$ fixed, in the second and the third terms of the right-hand side of (4.14). By Lemma 4.2, we deduce that there exists $\xi_{0} \in\left(L_{\bar{\varphi}}(\Omega)\right)^{N}$ and 
up to a subsequence $a\left(x, T_{n}\left(u_{n}\right), \nabla u_{n}\right) \rightarrow \xi_{0}$ weakly in $\left(L_{\bar{\varphi}}(\Omega)\right)^{N}$ for $\sigma\left(\Pi L_{\bar{\varphi}}, \Pi E_{\varphi}\right)$. Since $\nabla v_{j} \chi_{\Omega \backslash \Omega_{j}^{s}} \in\left(E_{\varphi}(\Omega)\right)^{N}$, we have by letting $n \rightarrow \infty$,

$$
-\int_{\Omega \backslash \Omega_{j}^{s}} a\left(x, T_{n}\left(u_{n}\right), \nabla u_{n}\right) \cdot \nabla v_{j} d x \rightarrow-\int_{\Omega \backslash \Omega_{j}^{s}} \xi_{0} \cdot \nabla v_{j} d x .
$$

Using the modular convergence of $v_{j}$, we get as $j \rightarrow \infty$

$$
-\int_{\Omega \backslash \Omega_{j}^{s}} \xi_{0} \cdot \nabla v_{j} d x \rightarrow-\int_{\Omega \backslash \Omega^{s}} \xi_{0} \cdot \nabla u d x .
$$

Hence, we have proved that the third term

$$
-\int_{\Omega \backslash \Omega_{j}^{s}} a\left(x, T_{n}\left(u_{n}\right), \nabla u_{n}\right) \cdot \nabla v_{j} d x=-\int_{\Omega \backslash \Omega^{s}} \xi_{0} \cdot \nabla u d x+\epsilon_{1}(n, j) .
$$

For the second term, as $n \rightarrow \infty$, we have

$$
\int_{\Omega} a\left(x, T_{n}\left(u_{n}\right), \nabla v_{j} \chi_{j}^{s}\right) \cdot\left(\nabla u_{n}-\nabla v_{j} \chi_{j}^{s}\right) d x \rightarrow \int_{\Omega} a\left(x, u, \nabla v_{j} \chi_{j}^{s}\right) \cdot\left(\nabla u-\nabla v_{j} \chi_{j}^{s}\right) d x,
$$

since $a\left(x, T_{n}\left(u_{n}\right), \nabla v_{j} \chi_{j}^{s}\right) \rightarrow a\left(x, u, \nabla v_{j} \chi_{j}^{s}\right)$ strongly in $\left(E_{\bar{\varphi}}(\Omega)\right)^{N}$ as $n \rightarrow \infty$ by lemma 2.2 and (4.11), while $\nabla u_{n} \rightarrow \nabla u$ weakly in $\left(L_{\varphi}(\Omega)\right)^{N}$ by (4.10). And since $\nabla v_{j} \chi_{j}^{s} \rightarrow \nabla u \chi^{s}$ strongly in $\left(E_{\varphi}(\Omega)\right)^{N}$ as $j \rightarrow \infty$, we obtain

$$
\int_{\Omega} a\left(x, u, \nabla v_{j} \chi_{j}^{s}\right) \cdot\left(\nabla u-\nabla v_{j} \chi_{j}^{s}\right) d x \rightarrow 0
$$

as $j \rightarrow \infty$. So that

$$
\int_{\Omega} a\left(x, T_{n}\left(u_{n}\right), \nabla v_{j} \chi_{j}^{s}\right) \cdot\left(\nabla u_{n}-\nabla v_{j} \chi_{j}^{s}\right) d x=\epsilon_{2}(n, j) .
$$

Then, from (4.13), (4.15) and (4.16), we obtain

$$
\begin{aligned}
& \int_{\Omega}\left(a\left(x, T_{n}\left(u_{n}\right), \nabla u_{n}\right)-a\left(x, T_{n}\left(u_{n}\right), \nabla v_{j} x_{j}^{s}\right)\right) \cdot\left(\nabla u_{n}-\nabla v_{j} \chi_{j}^{s}\right) d x \\
& =\int_{\Omega \backslash \Omega^{s}} \xi_{0} \cdot \nabla u d x+\epsilon_{3}(n, j) .
\end{aligned}
$$

On the other hand

$$
\begin{aligned}
& \int_{\Omega}\left(a\left(x, T_{n}\left(u_{n}\right), \nabla u_{n}\right)-a\left(x, T_{n}\left(u_{n}\right), \nabla u \chi^{s}\right)\right) \cdot\left(\nabla u_{n}-\nabla u \chi^{s}\right) d x \\
& =\int_{\Omega}\left(a\left(x, T_{n}\left(u_{n}\right), \nabla u_{n}\right)-a\left(x, T_{n}\left(u_{n}\right), \nabla v_{j} \chi_{j}^{s}\right)\right) \cdot\left(\nabla u_{n}-\nabla v_{j} x_{j}^{s}\right) d x \\
& \quad+\int_{\Omega} a\left(x, T_{n}\left(u_{n}\right), \nabla u_{n}\right) \cdot\left(\nabla v_{j} \chi_{j}^{s}-\nabla u \chi^{s}\right) d x \\
& \quad-\int_{\Omega} a\left(x, T_{n}\left(u_{n}\right), \nabla u \chi^{s}\right) \cdot\left(\nabla u_{n}-\nabla u \chi^{s}\right) d x \\
& \quad+\int_{\Omega} a\left(x, T_{n}\left(u_{n}\right), \nabla v_{j} \chi_{j}^{s}\right) \cdot\left(\nabla u_{n}-\nabla v_{j} \chi_{j}^{s}\right) d x .
\end{aligned}
$$


Proceeding as above, we obtain

$$
\begin{aligned}
& \int_{\Omega} a\left(x, T_{n}\left(u_{n}\right), \nabla u_{n}\right) \cdot\left(\nabla v_{j} \chi_{j}^{s}-\nabla u \chi^{s}\right) d x=\epsilon_{4}(n, j), \\
& \int_{\Omega} a\left(x, T_{n}\left(u_{n}\right), \nabla u \chi^{s}\right) \cdot\left(\nabla u_{n}-\nabla u \chi^{s}\right) d x=\epsilon_{5}(n, j), \\
& \int_{\Omega} a\left(x, T_{n}\left(u_{n}\right), \nabla v_{j} \chi_{j}^{s}\right) \cdot\left(\nabla u_{n}-\nabla v_{j} \chi_{j}^{s}\right) d x=\epsilon_{6}(n, j) .
\end{aligned}
$$

Then, by (4.17) we have

$$
\begin{aligned}
& \int_{\Omega}\left(a\left(x, T_{n}\left(u_{n}\right), \nabla u_{n}\right)-a\left(x, T_{n}\left(u_{n}\right), \nabla u \chi^{s}\right)\right) \cdot\left(\nabla u_{n}-\nabla u \chi^{s}\right) d x \\
& =\epsilon_{7}(n, j)+\int_{\Omega \backslash \Omega^{s}} \xi_{0} \cdot \nabla u d x .
\end{aligned}
$$

For $r \leq s$, we write

$$
\begin{aligned}
0 & \leq \int_{\Omega^{r}}\left(a\left(x, T_{n}\left(u_{n}\right), \nabla u_{n}\right)-a\left(x, T_{n}\left(u_{n}\right), \nabla u\right)\right) \cdot\left(\nabla u_{n}-\nabla u\right) d x \\
& \leq \int_{\Omega^{s}}\left(a\left(x, T_{n}\left(u_{n}\right), \nabla u_{n}\right)-a\left(x, T_{n}\left(u_{n}\right), \nabla u\right)\right) \cdot\left(\nabla u_{n}-\nabla u\right) d x \\
& =\int_{\Omega^{s}}\left(a\left(x, T_{n}\left(u_{n}\right), \nabla u_{n}\right)-a\left(x, T_{n}\left(u_{n}\right), \nabla u \chi^{s}\right)\right) \cdot\left(\nabla u_{n}-\nabla u \chi^{s}\right) d x \\
& \leq \int_{\Omega}\left(a\left(x, T_{n}\left(u_{n}\right), \nabla u_{n}\right)-a\left(x, T_{n}\left(u_{n}\right), \nabla u \chi^{s}\right)\right) \cdot\left(\nabla u_{n}-\nabla u \chi^{s}\right) d x \\
& \leq \epsilon_{7}(n, j)+\int_{\Omega \backslash \Omega^{s}} \xi_{0} \cdot \nabla u d x .
\end{aligned}
$$

Passing at first to the limit superior over $n$ and then over $j$, it follows

$$
\begin{aligned}
0 & \leq \limsup _{n \rightarrow \infty} \int_{\Omega^{r}}\left(a\left(x, T_{n}\left(u_{n}\right), \nabla u_{n}\right)-a\left(x, T_{n}\left(u_{n}\right), \nabla u\right)\right) \cdot\left(\nabla u_{n}-\nabla u\right) d x \\
& \leq \int_{\Omega \backslash \Omega^{s}} \xi_{0} \cdot \nabla u d x .
\end{aligned}
$$

Letting $s \rightarrow+\infty$ in the previous inequality, we conclude that as $n \rightarrow \infty$,

$$
\int_{\Omega^{r}}\left(a\left(x, T_{n}\left(u_{n}\right), \nabla u_{n}\right)-a\left(x, T_{n}\left(u_{n}\right), \nabla u\right)\right) \cdot\left(\nabla u_{n}-\nabla u\right) d x \rightarrow 0
$$

Define $A_{n}$ by

$$
A_{n}=\left(a\left(x, T_{n}\left(u_{n}\right), \nabla u_{n}\right)-a\left(x, T_{n}\left(u_{n}\right), \nabla u\right)\right) \cdot\left(\nabla u_{n}-\nabla u\right)
$$

As a consequence of (4.18), one has $A_{n} \rightarrow 0$ strongly in $L^{1}\left(\Omega^{r}\right)$, extracting a subsequence, still denoted by $\left\{u_{n}\right\}$, we get $A_{n} \rightarrow 0$ a.e in $\Omega^{r}$. Then, there exists a subset $\Omega_{0}$ of $\Omega^{r}$, of zero measure, such that: $A_{n}(x) \rightarrow 0$ for all $x \in \Omega^{r} \backslash \Omega_{0}$. On the other hand, using (3.2), (3.3) and 
then lemma 3.1 we obtain for all $x \in \Omega^{r} \backslash \Omega_{0}$,

$$
\begin{aligned}
A_{n}(x) \geq & \bar{\varphi}_{x}^{-1}\left(\varphi\left(x, h\left(c_{\infty}\right)\right)\right) \varphi\left(x,\left|\nabla u_{n}(x)\right|\right) \\
& -c_{1}(x)\left(1+\bar{\varphi}_{x}^{-1}\left(\varphi\left(x, k_{4}\left|\nabla u_{n}(x)\right|\right)\right)+\left|\nabla u_{n}(x)\right|\right) \\
\geq & \bar{q}^{-1}\left(q\left(h\left(c_{\infty}\right)\right)\right) \varphi\left(x,\left|\nabla u_{n}(x)\right|\right) \\
& -c_{1}(x)\left(1+\bar{\varphi}_{x}^{-1}\left(\varphi\left(x, k_{4}\left|\nabla u_{n}(x)\right|\right)\right)+\left|\nabla u_{n}(x)\right|\right)
\end{aligned}
$$

where $c_{\infty}$ is the constant which appears in (4.5) and $c_{1}(x)$ is a constant which does not depend on $n$. For $x$ fixed the sequence $\left\{\nabla u_{n}(x)\right\}$ is bounded in $\mathbb{R}^{N}$, else, if $\left\{\nabla u_{n}(x)\right\}$ is unbounded, there exists a subsequence still denoted by $\left\{\nabla u_{n}(x)\right\}$ which tends to $+\infty$ and we have $\varphi\left(x,\left|\nabla u_{n}(x)\right|\right) \rightarrow+\infty$ and recall the inequality

$$
\varphi(x, t) \leq t \bar{\varphi}_{x}^{-1}(\varphi(x, t)) \leq 2 \varphi(x, t),
$$

for all $t \geq 0$, which implies that

$$
\bar{\varphi}_{x}^{-1}\left(\varphi\left(x, k_{4}\left|\nabla u_{n}(x)\right|\right)\right) \leq \frac{2 \varphi\left(x, k_{4}\left|\nabla u_{n}(x)\right|\right)}{k_{4}\left|\nabla u_{n}(x)\right|} .
$$

Since $\varphi$ satisfies the $\Delta_{2}$-condition, there exists a positive constant $k$ and a positive $L^{1}$-function $c$ such that

$$
\bar{\varphi}_{x}^{-1}\left(\varphi\left(x, k_{4}\left|\nabla u_{n}(x)\right|\right)\right) \leq \frac{2 \varphi\left(x, k_{4}\left|\nabla u_{n}(x)\right|\right)}{k_{4}\left|\nabla u_{n}(x)\right|} \leq \frac{2 k \varphi\left(x,\left|\nabla u_{n}(x)\right|\right)}{k_{4}\left|\nabla u_{n}(x)\right|}+c(x) .
$$

Consequently, the right term in the inequality (4.19) goes to $+\infty$ as $n$ goes to $+\infty$, which is a contradiction with $\lim _{n \rightarrow \infty} A_{n}=0$. Thus, $\left\{\nabla u_{n}(x)\right\}$ is bounded, then for a subsequence $\left\{u_{n^{\prime}}(x)\right\}$, we have

$$
\begin{gathered}
\nabla u_{n^{\prime}}(x) \rightarrow \xi \quad \text { in } \mathbb{R}^{N} \\
(a(x, u(x), \xi)-a(x, u(x), \nabla u(x))) \cdot(\xi-\nabla u(x))=0 .
\end{gathered}
$$

Since $a(x, s, \xi)$ is strictly monotone, we have $\xi=\nabla u(x)$, and so $\nabla u_{n}(x) \rightarrow \nabla u(x)$ for the whole sequence. It follows that

$$
\nabla u_{n} \rightarrow \nabla u \quad \text { a.e. in } \Omega^{r} \text {. }
$$

Consequently, as $r$ is arbitrary, we deduce

$$
\nabla u_{n} \rightarrow \nabla u \quad \text { a.e. in } \Omega \text {. }
$$

Step 6: Passing to the limit. Let $v$ be a function in $\mathfrak{D}(\Omega)$. Taking $v$ as test function in (4.1), one has

$$
\int_{\Omega} a\left(x, T_{n}\left(u_{n}\right), \nabla u_{n}\right) \cdot \nabla v d x=\int_{\Omega} f_{n} v d x .
$$

Lemma 4.2, (4.11) and (4.20) imply that

$$
a\left(x, T_{n}\left(u_{n}\right), \nabla u_{n}\right) \rightarrow a(x, u, \nabla u) \quad \text { weakly in }\left(L_{\bar{\varphi}}(\Omega)\right)^{N} \text { for } \sigma\left(\Pi L_{\bar{\varphi}}, \Pi E_{\varphi}\right),
$$


so, passing to the limit in the previous equality to obtain

$$
\int_{\Omega} a(x, u, \nabla u) \cdot \nabla v d x=\int_{\Omega} f v d x .
$$

Moreover, from (4.5) and (4.11) we have $u \in W_{0}^{1} L_{\varphi}(\Omega) \cap L^{\infty}(\Omega)$. This completes the proof of theorem 3.1.

\section{References}

[1] R. Adams; Sobolev spaces, Academic Press Inc, New York, (1975).

[2] Y. Ahmida, I. Chlebicka, P. Gwiazda, A. Youssfi, Gossez's approximation theorems in Musielak-Orlicz-Sobolev spaces, J. Funct. Anal. (2018), https://doi.org/10.1016/j.jfa.2018.05.015

[3] Y. Ahmida, A. Youssfi, Poincaré- type inequalities in Musielak spaces, Annales Academi Scientiarum Fennic Mathematica, (2019), https:/ / doi.org/10.5186/aasfm.2019.4468

[4] A. Alvino, L. Boccardo, V. Ferone, L. Orsina, G. Trombetti; Existence results for nonlinear elliptic equations with degenerate coercivity, Ann. Mat. Pura Appl., IV. Ser. 182, No.1, (2003), 53-79.

[5] M. Ait Khellou, A. Benkirane, S.M. Douiri; Existence of solutions for elliptic equations having natural growth terms in Musielak-Orlicz spaces. J. Math. Comput. Sci. 4(4), 665688 (2014)

[6] A. Alvino, V. Ferone, G. Trombetti; A priori estimates for a class of non uniformly elliptic equations, Atti Semin. Mat. Fis. Univ. Modena 46-suppl., (1998), 381-391.

[7] A. Benkirane, M. Sidi El Vally(Ould Mohameden Val); Variational inequalities in Musielak-Orlicz-Sobolev spaces, Bull. Belg. Math.Soc. Simon Stevin, Vol 21, No.5 (2014), pp. 787-811.

[8] A. Benkirane, M. Sidi El Vally(Ould Mohameden Val); An existence result for nonlinear elliptic equations in Musielak-Orlicz-Sobolev spaces, Bull. Belg. Math.Soc. Simon Stevin 20(2013), No. 1, 57-75.

[9] L. Boccardo, A. Dall'Aglio, L. Orsina; Existence and regularity results for some elliptic equations with degenerate coercivity. Atti Semin. Mat. Fis. Univ. Modena 46-suppl., (1998), 51-81.

[10] M. Bourahma, A. Benkirane, J. Bennouna, Existence of renormalized solutions for some nonlinear elliptic equations in Orlicz spaces, J. Rend. Circ. Mat. Palermo, II. Ser (2019). https://doi.org/10.1007/s12215-019-00399-z

[11] I. Chlebicka, P. Gwiazda and A. Zatorska Goldstein, Well-posedness of parabolic equations in the non-reflexive and anisotropic Musielak-Orlicz spaces in the class of renormalized solutions, arXiv:1707.06097v4 [math.AP] 13 Apr 2018.

[12] J. P. Gossez; A strongly nonlinear elliptic problem in Orlicz-Sobolev spaces, Proc. Sympos. Pure Math. 45, Amer. Math. Soc., (1986), 455-462.

[13] J. P. Gossez, Nonlinear elliptic boundary value problems for equations with rapidly (or slowly) increasing coefficients, Trans. Amer. Math. soc. 190, (1974), 163-205.

[14] P. Gwiazda, I. Skrzypczak, A. Zatorska.Goldstein, Existence of renormalized solutions to elliptic equation in Musielak-Orlicz space, J. Differential Equations 264 (2018) 341377.

[15] M. Krasnosel'skii, Ya. Rutikii; Convex functions and Orlicz spaces, Groningen, Nordhooff (1969).

[16] J. Musielak; Orlicz spaces and modular spaces, Lecture Note in Mahtematics, 1034, Springer, Berlin, 1983.

[17] G. Talenti; Nonlinear elliptic equations, Rearrangements of functions and Orlicz spaces, Ann. Mat. Pura Appl., IV. Ser. 120, (1979), 159-184.

[18] G. Talenti; Elliptic equations and rearrangements, Ann. Scuola Norm. Sup. Pisa (4) 3, (1976), 697-718.

[19] A. Youssfi; Existence of bounded solution for nonlinear degenerate elliptic equations in Orlicz spaces, Electronic Journal of Differential Equations, Vol. 2007(2007), No. 54, pp. 1-13. 